\section{Situación actual del trasplante hepático pediátrico por hepatopatías crónicas en Chile: Inequidades asociadas a la priorización por sistema MELD/PELD}

\author{
LUIS ANTONIO DÍAZ*1, MARISOL LÓPEZ*2, PRISCILA SIN ${ }^{3}$, \\ RODRIGO WOLFF ${ }^{1}$, GLORIA GONZÁLEZ ${ }^{4}$, MARÍA PAZ MUÑOZ ${ }^{4}$, \\ MARIO URIBE ${ }^{4}$, ÁLVARO ANANIAS ${ }^{5}$, IGNACIO BEZAMA ${ }^{5}$, \\ NICOLÁS ZAÑARTU ${ }^{5}$, ERWIN BUCKEL ${ }^{6}$, FRANCO INNOCENTI ${ }^{7}$, \\ JUAN CARLOS PATTILLO ${ }^{8}$, NICOLÁS JARUFE ${ }^{6}$, \\ JORGE MARTÍNEZ ${ }^{8}$, JUAN FRANCISCO GUERRA ${ }^{9}$, \\ SUSANA ELGUETA ${ }^{10}$, JUAN CRISTÓBAL GANA ${ }^{3}$
}

\section{Current situation of pediatric liver} transplantation in Chile. Inequities associated
with the MELD/PELD prioritization system

Background: The Chilean allocation system for liver transplantation (LT) uses the MELD/PELD score to prioritize candidates on the waiting list. Aim: To assess if the Chilean allocation system for $L T$ is equitable for pediatric candidates compared to their adult counterparts. Material and Methods: We used the Public Health Institute's registry between October 2011 and December 2017. We analyzed candidates with chronic hepatic diseases listed for LT. The primary outcome was the cadaveric liver transplantation (CLT) rate. Secondary outcomes were death or disease progression in the waiting list and living donor liver transplant (LDLT) rate. Results: We analyzed 122 pediatric and 735 adult candidates. Forty one percent of pediatric candidates obtained a CLT compared to $48 \%$ of adults $(p=N S)$. Among patients aged under two years of age, the access to CLT on the waiting list there was $28 \%$ of CLT, compared to $48 \%$ in adults $(p=0.001)$. Fifty-seven percent of candidates aged under two years were listed for cholestatic diseases, obtaining a CLT in $18 \%$ and requiring a LDLT in $49 \%$. The median time in the waiting list for CLT was 5.9 months in pediatric candidates and 5.1 in adults, while the median time to death in the waiting list was 2.8 and 5.6 months, respectively. The mortality rate at one year in candidates under two years old was $38.1 \%$ compared to $32.5 \%$ in adults. Conclusions: Pediatric candidates with chronic liver diseases, especially under two years of age, have greater access difficulties to CLT than adults. Half of the pediatric candidates die on the waiting list before three months. The mortality among candidates under two years of age in the waiting list is excessively high.

(Rev Med Chile 2020; 148: 1261-1270)

Key words: Liver Diseases; Living Donors; Liver Transplantation; Resource Allocation; Waiting Lists.
'Departamento de

Gastroenterología, Pontificia Universidad Católica de Chile. Santiago, Chile.

'Departamento de Pediatría, Pontificia Universidad Católica de Chile. Santiago, Chile.

${ }^{3}$ Departamento de

Gastroenterología y Nutrición Pediátrica, División de Pediatría. Escuela de Medicina. Pontificia Universidad Católica de Chile.

Santiago, Chile.

${ }^{4}$ Hospital Dr. Luis Calvo

Mackenna. Santiago, Chile.

${ }^{5}$ Escuela de Medicina, Pontificia Universidad Católica de Chile.

Santiago, Chile.

${ }^{6}$ Clínica Las Condes. Santiago, Chile.

${ }^{7}$ Clínica Sanatorio Alemán. Concepción, Chile.

${ }^{8}$ División de Cirugía, Escuela de Medicina, Pontificia Universidad Católica de Chile. Santiago, Chile. ${ }^{9}$ MedStar Georgetown Transplant Institute, Medstar Georgetown University Hospital. Washington D.C., Estados Unidos de Norteamérica.

${ }^{10}$ Instituto de Salud Pública.

Santiago, Chile.

*Estos autores comparten la primera autoría.

Trabajo no recibió financiamiento. Los autores declaran no tener conflictos de interés.

Recibido el 26 de marzo de 2020, aceptado el 14 de septiembre de 2020.

Correspondencia a:

Dr. Juan Cristóbal Gana Ansaldo Departamento de Gastroenterología y Nutrición Pediátrica, Pontificia Universidad Católica de Chile.

Diagonal Paraguay 362, Santiago Centro. Santiago, Chile. jcgana@gmail.com 
$\mathrm{E}$ n Chile, se producen cerca de 3.000 muertes al año por enfermedades hepáticas, y en la mayoría de estos casos la única opción terapéutica es el trasplante hepático $(\mathrm{TH})^{1}$. Hasta octubre de 2011, la asignación de hígados de donante cadáver (DC) en Chile se basaba en el carácter de urgencia y la antigüedad en lista de espera (LE) de los candidatos, alcanzando una mortalidad en LE por hepatopatías crónicas de 25\%-29\% $\%^{2,3}$. Posteriormente, el criterio de antigüedad fue reemplazado por el de gravedad, mediante puntajes MELD y PELD (Model for End Stage Liver Disease y Pediatric End Liver Disease $)^{4,5}$. En un estudio chileno previo se observó una tasa de TH con donante cadáver (THDC) de 43,3\%, con una mediana de tiempo de espera al TH de 4,25 meses y una mortalidad en LE al año de $24,76 \%{ }^{6}$. Respecto a los pacientes pediátricos alistados en similares condiciones en nuestro país, no existen datos publicados.

Los sistemas de puntaje MELD/PELD son ampliamente usados en muchos centros de $\mathrm{TH}$ del mundo. En Argentina, por ejemplo, tras la incorporación de este sistema de puntaje, se redujo la mortalidad en LE a 3 meses de $32 \%$ a $18 \%{ }^{7}$. No obstante, Latinoamérica se encuentra lejos de cifras de países desarrollados, como las de Estados Unidos de Norteamérica, en que la mortalidad de candidatos pediátricos en LE es menor a $10 \%{ }^{8}$.

Aunque se han instaurado importantes cambios en la priorización de pacientes con hepatopatías crónicas menores de 15 años en el contexto de una baja tasa de donantes en Chile, no existen datos publicados acerca de la situación actual del sistema de priorización. Creemos importante analizar la equidad en la asignación de injertos cadavéricos entre candidatos pediátricos y adultos, dadas las diferencias en las etiologías de alistamiento, en las causas de excepciones y la proporción de pacientes que dejan la LE por no lograr recibir un injerto, en ambos grupos. El objetivo de este estudio es conocer la situación actual del TH en pacientes ingresados al programa por daño hepático crónico, con énfasis en la mortalidad en LE, y el acceso oportuno a un THDC, para evaluar la equidad en la asignación entre pacientes pediátricos y adultos.

\section{Materiales y Métodos}

Examinamos el registro del Instituto de Salud Pública de Chile (ISP) de candidatos alistados para $\mathrm{TH}$, la información utilizada fue autorizada por el
Coordinador Nacional de Trasplante. Adicionalmente, este estudio fue aprobado por el Comité de Ética de la Pontificia Universidad Católica de Chile (ID 170315009).

Analizamos los datos de candidatos a TH, alistados desde el 15 de octubre de 2011 (inicio de la priorización por sistema MELD/PELD en Chile) hasta el 31 de diciembre de 2017, con seguimiento de supervivencia hasta el 30 de junio de 2018. Incluimos a los candidatos alistados para TH por patologías crónicas. Excluimos a candidatos alistados en calidad de urgencia, candidatos con contraindicaciones médicas o quirúrgicas, candidatos que perdieron controles ambulatorios, pacientes pediátricos retirados de LE por decisión de sus tutores legales y adultos retirados de LE por consumo activo de sustancias (Figura 1).

Se usaron datos desidentificados entregados por el ISP. Analizamos los datos dividiendo a los pacientes en 3 grupos etarios: de recién nacido a 1 año 11 meses 29 días, de 2 años a 14 años 11 meses 29 días y de 15 años o más. Los datos analizados fueron, las etiologías de alistamiento, el motivo de retiro de LE (THDC, TH donante vivo [DV] o fallecimiento en LE) y el tiempo de espera hasta este retiro.

\section{Análisis estadístico}

Los datos continuos se describieron utilizando media y desviación estándar (DE), o mediana y rangos intercuartiles (IQ) (entre los percentiles $25 \%$ y $75 \%$ ) por distribución de datos. Los datos nominales se describieron utilizando porcentajes. Se consideró el puntaje MELD/PELD operacional, que es el puntaje real en aquellos pacientes con hepatopatías crónicas sin excepciones y el puntaje real más puntos extras en aquellos pacientes con excepción. Se utilizaron estimaciones de supervivencia mediante curvas de Kaplan-Meier y comparaciones mediante log-rank. Las diferencias en las proporciones se probaron utilizando la prueba de chi-cuadrado y la prueba U Mann-Whitney. El análisis estadístico se realizó con el software IBM SPSS versión 22.0 (IBM Corp. Armonk, NY, USA) y el software STATA versión 13.0 (StataCorp; College Station, TX, USA). Se consideró significancia con un valor $\mathrm{p}<0,05$.

\section{Resultados}

Se alistaron 1.052 candidatos para $\mathrm{TH}$ en el periodo descrito (Figura 1). No hubo diferencias 


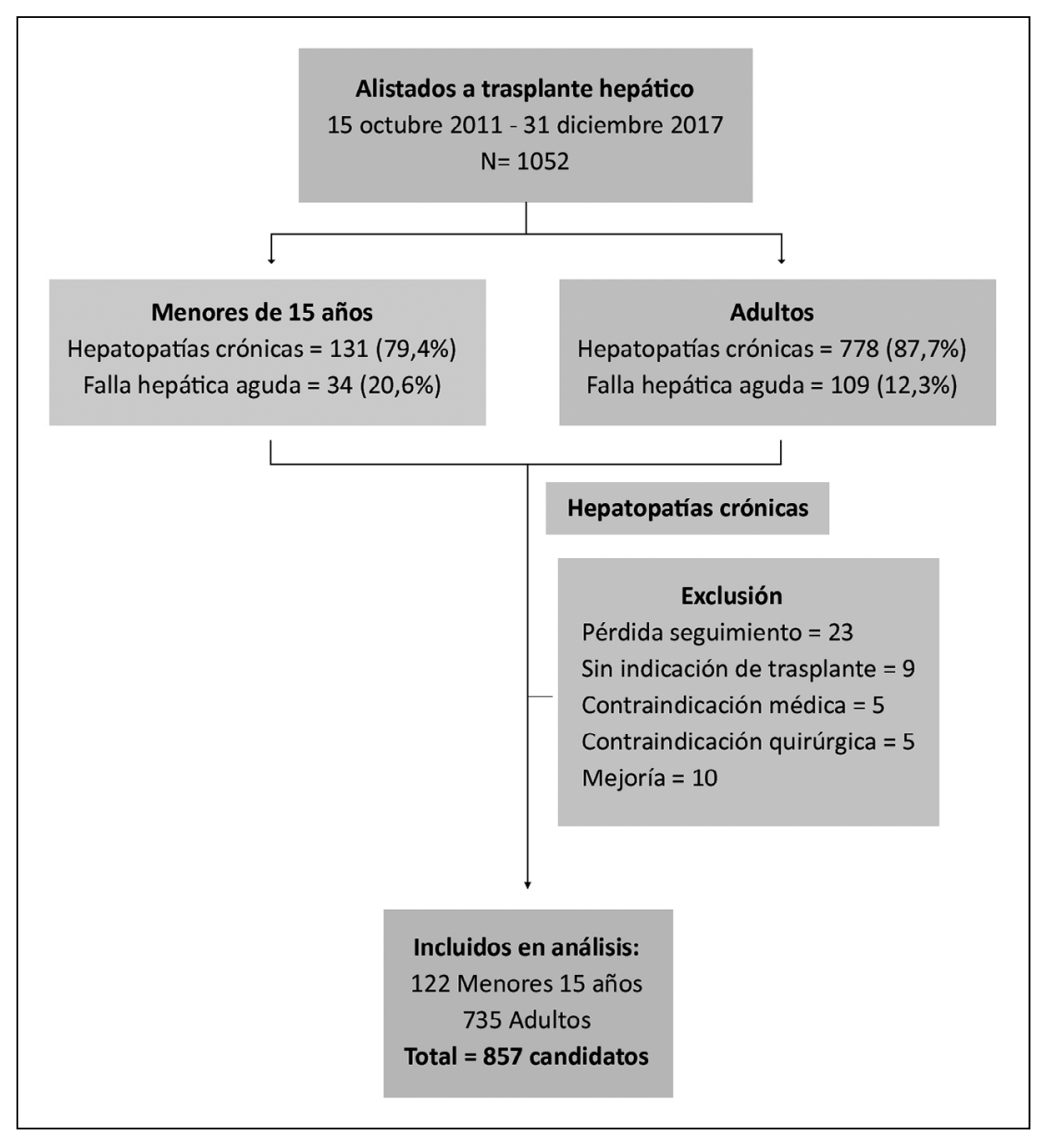

Figura 1. Criterios de exclusión en el análisis de candidatos alistados por hepatopatías crónicas.

Tabla 1. Distribución de pacientes incluidos por grupo etario, mediana de edad y mediana de puntaje MELD/PELD al alistamiento

\begin{tabular}{|lccc|}
\hline Grupo etario & $\begin{array}{c}\text { Incluidos } \\
\mathbf{n ~ ( \% )}\end{array}$ & $\begin{array}{c}\text { Edad } \\
\text { Mediana [IQ 25-75] }\end{array}$ & $\begin{array}{c}\text { MELD/PELD ingreso a lista } \\
\text { Mediana [IQ 25-75] }\end{array}$ \\
\hline Menores de 15 años & $122(14,3)$ & 1,1 años $[0,6-5,8$ años] & $21[15-25]$ \\
Menores de 2 años & 68 & 8,4 meses $[6-12$ meses] & $21[19-25]$ \\
2 años a 15 años & 54 & 6,14 años [3,3-11,1 años] & $19[15-25]$ \\
Mayores 15 años & $730(86,7)$ & 57 años $[49-62,7$ años] & $20[16-22]$ \\
\hline
\end{tabular}

significativas en la mediana de puntaje MELD/ PELD al alistamiento en cada grupo $(\mathrm{p}=0,136)$ (Tabla 1). La mediana de puntaje MELD/PELD al alistamiento fue menor en pacientes con etiología colestásica comparado con otras etiologías (17 [9-21] versus 21 [20-30], respectivamente; $\mathrm{p}=0,003)$. Debido a falta de registro del puntaje MELD/PELD al retiro de LE (por trasplante o fallecimiento), no se consideró esta variable en el análisis. En la población pediátrica, $57,1 \%$ de los pacientes se alistó con excepciones: $58,5 \%$ en el caso de los menores de 2 años y $56,6 \%$ en los candidatos entre 2 y 15 años.

\section{Etiologías de alistamiento}

Las principales etiologías en candidatos pediátricos fueron la atresia de vía biliar $(41,8 \%)$, el hepatoblastoma $(7,4 \%)$ y la enfermedad poli- 
Tabla 2. Causas de alistamiento para trasplante hepático en candidatos pediátricos con hepatopatías crónicas, según grupo etario

\begin{tabular}{|lccc|}
\hline Causa & $\begin{array}{c}<\mathbf{2} \text { años } \\
\text { n (\%) }\end{array}$ & $\begin{array}{c}\mathbf{2} \text { a } \mathbf{1 5} \text { años } \\
\text { n (\%) }\end{array}$ & $\begin{array}{c}\text { Total menores 15 años } \\
\text { n (\%) }\end{array}$ \\
Colestasia & $39(57,4)$ & $22(40,7)$ & $61(50)$ \\
Atresia vía biliar & $34(50)$ & $17(31,5)$ & $51(41,8)$ \\
Síndrome de Alagille & $2(2,9)$ & $4(7,4)$ & $6(4,9)$ \\
Otras & $3(4,4)$ & $1(1,9)$ & $4(3,3)$ \\
Tumores & $3(4,4)$ & $8(14,8)$ & $11(9)$ \\
Hepatoblastoma & $3(4,4)$ & $6(11,1)$ & $9(7,4)$ \\
Hepatocarcinoma & $0(0)$ & $2(3,7)$ & $2(1,6)$ \\
Enfermedad poliquística hepatorrenal & $0(0)$ & $9(16,7)$ & $9(7,4)$ \\
Errores innatos del metabolismo & $3(4,4)$ & $3(5,6)$ & $6(4,9)$ \\
Retrasplante tardío & $0(0)$ & $5(9,3)$ & $5(4,1)$ \\
Hepatitis autoinmune & $0(0)$ & $3(5,6)$ & $3(2,5)$ \\
Otros & $1(1,5)$ & $1(1,9)$ & $2(1,6)$ \\
Hemocromatosis neonatal & $1(1,5)$ & $0(0)$ & $1(0,8)$ \\
Fibrosis quística & $0(0)$ & $1(1,9)$ & $1(0,8)$ \\
No precisado & $22(32,3)$ & $3(5,6)$ & $25(20,5)$ \\
\hline Total & 68 & 54 & 122 \\
\hline
\end{tabular}

quística hepatorrenal (7,4\%) (Tabla 2). En el caso de los adultos, las principales etiologías fueron la esteatohepatitis no alcohólica $(27,7 \%)$ y alcohol $(15,2 \%)$ (Tabla 3$)$.

Análisis del funcionamiento de la lista de espera

En el período se realizaron $50(41 \%)$ THDC en candidatos pediátricos y $351(47,8 \%)$ en adultos $(\mathrm{p}=0,098)($ Tabla 4$)$. El subgrupo de menores de 2 años requirió un THDV en $33(48,5 \%)$ candidatos y $15(22,1 \%)$ fueron retirados por muerte o progresión, logrando acceso a THDC solo en $27,9 \%$. Seis $(0,8 \%)$ candidatos adultos accedieron a THDV y $273(37,1 \%)$ fueron retirados de lista de espera por muerte o progresión, con un acceso a THDC de 47,8\% (valor $\mathrm{p}=0,001$ al comparar menores de 2 años con adultos).

\section{Tiempos de espera}

Al comparar el tiempo en LE, no hubo diferencias en la espera para THDC entre pacientes pediátricos y adultos $(5,9[2,1-14,4]$ meses versus $5,1[1,1-13,6]$ meses, respectivamente; $p=0,535)$,
Tabla 3. Causas de alistamiento para trasplante hepático en adultos con hepatopatías crónicas

\begin{tabular}{|lrc|}
\hline Causa & \multicolumn{3}{c|}{$\begin{array}{c}\text { Adultos } \\
\text { n }\end{array}$} & $\begin{array}{l}(\%) \\
\text { Esteatohepatitis no alcohólica }\end{array}$ & 202 & $(27,7)$ \\
\hline Alcohol & 111 & $(15,2)$ \\
\hline Hepatitis autoinmune & 81 & $(11,1)$ \\
Hepatitis virales & 75 & $(10,3)$ \\
Virus hepatitis C & 72 & $(9,9)$ \\
Virus hepatitis B & 3 & $(0,4)$ \\
Enfermedades colestásicas & 57 & $(7,8)$ \\
Colangitis biliar primaria & 31 & $(4,2)$ \\
Síndrome de sobreposición & 16 & $(2,2)$ \\
Colangitis esclerosante primaria & 8 & $(1,1)$ \\
Cirrosis biliar secundaria & 2 & $(0,3)$ \\
Otros & 145 & $(19,9)$ \\
No precisado & 59 & $(8)$ \\
Total & 730 & $(100)$ \\
\hline
\end{tabular}


Tabla 4. Funcionamiento de lista de espera según grupo etario

\begin{tabular}{|ccccc|}
\hline & Aún en lista & THDC & THDV & F/P \\
& & $\mathbf{n}(\mathbf{\%})$ & $\mathbf{n}(\%)$ & $15(22,1)$ \\
\hline 2 años & $1(1,5)$ & $19(27,9)$ & $33(48,5)$ & $6(11,1)$ \\
\hline 2 a 15 años & $11(20,4)$ & $31(57,4)$ & $6(11,1)$ & $273(37,1)$ \\
\hline 15 años & $105(14,3)$ & $351(47,8)$ & $6(0,8)$ & $294(34,3)$ \\
\hline Total & $117(13,6)$ & $401(46,8)$ & $45(5,3)$ & (\%) \\
\hline
\end{tabular}

THDC: trasplante hepático donante cadáver; THDV: trasplante hepático donante vivo; F/P: candidatos retirados de lista de espera por fallecimiento o progresión de la enfermedad.

Tabla 5. Funcionamiento LE por causa de alistamiento colestásica o no colestásica en los diferentes grupos etarios

\begin{tabular}{|c|c|c|c|c|c|c|c|c|}
\hline & \multicolumn{4}{|c|}{ Colestasia } & \multicolumn{4}{|c|}{ No colestasia } \\
\hline & $\begin{array}{l}\text { THDC } \\
\text { n }(\%)\end{array}$ & $\begin{array}{l}\text { THDV } \\
\text { n (\%) }\end{array}$ & $\begin{array}{c}\text { F/P } \\
n \quad(\%)\end{array}$ & $\begin{array}{c}\text { Aún en lista } \\
n(\%)\end{array}$ & $\begin{array}{l}\text { THDC } \\
\text { n (\%) }\end{array}$ & $\begin{array}{l}\text { THDV } \\
\text { n }(\%)\end{array}$ & $\begin{array}{c}\text { F/P } \\
\text { n }(\%)\end{array}$ & $\begin{array}{c}\text { Aún en lista } \\
n(\%)\end{array}$ \\
\hline Menores 2 años & $7(17,9)$ & $21(53,9)$ & $10(25,6)$ & $1(2,6)$ & $12(41,4)$ & $12(41,4)$ & $5(17,2)$ & $0 \quad(0)$ \\
\hline 2 a 15 años & $5(22,7)$ & $5(22,7)$ & $4(18,2)$ & $8(36,4)$ & $26(81,2)$ & $1(3,1)$ & $2(6,3)$ & $3(9,4)$ \\
\hline Mayores 15 años & $29(50,9)$ & $0 \quad(0)$ & $21(36,8)$ & $7(12,3)$ & $322(47,5)$ & $6(0,9)$ & $252(37,2)$ & $98(14,4)$ \\
\hline
\end{tabular}

THDC: trasplante hepático donante cadáver; THDV: trasplante hepático donante vivo; F/P: candidatos retirados de lista de espera por fallecimiento o progresión de la enfermedad.

en el tiempo de espera a THDV $(1,5[0,8-3,8]$ meses versus 3,0 [2,1-22,4] meses, respectivamente; $\mathrm{p}=0,566)$, ni en el tiempo al retiro de lista de espera $(2,8[1,0-5,8]$ meses versus 5,6 [2,1-12,3] meses, respectivamente; $\mathrm{p}=0,570)$.

\section{Análisis de la lista según causa colestásica y no colestásica}

Las etiologías colestásicas fueron más prevalentes en candidatos pediátricos que en adultos ( $50 \%$ vs $7,8 \%$, respectivamente), especialmente en menores de 2 años (57,4\% en menores de 2 años versus $40,7 \%$ en mayores de 2 años). Al evaluar el funcionamiento de la LE en este grupo específico, se observó que $79,5 \%$ de los menores de 2 años no lograron acceder a un THDC (Tabla 5).

\section{Supervivencia en lista de espera}

La mortalidad global a un año de los pacientes pediátricos fue $25,1 \%$. Al estratificar en los subgrupos menores 2 años, 2-15 años y adultos se observó una mortalidad estimada a un año de $38,1 \%, 14,3 \%$ y $32,5 \%$, respectivamente $(\mathrm{p}=0,006)$ (Figura 2). También se comparó la tasa de salida de lista (muerte o progresión de enfermedad tumoral), observando una salida de lista al año estimada de $41,3 \%, 14,3 \%$ y $45,1 \%$, respectivamente $(\mathrm{p}=0,002)$ (Figura 3$)$.

\section{Supervivencia posterior al trasplante}

La supervivencia global estimada en candidatos pediátricos postrasplante fue $91,7 \%$ a 1 año, la cual se mantuvo de forma consistente a 5 años. Al estratificar en subgrupos, la supervivencia postrasplante al año en menores 2 años, 2-15 años y adultos fue $87,6 \%, 97,2 \%$ y $86,1 \%$, respectivamente $(\mathrm{p}=0,136)$ (Figura 4). No se observaron diferencias en la supervivencia a 1 y 5 años entre candidatos pediátricos trasplantados con DC versus DV (91,7\% versus $91,7 \%, \mathrm{p}=0,944)(\mathrm{Fi}-$ gura 5). 


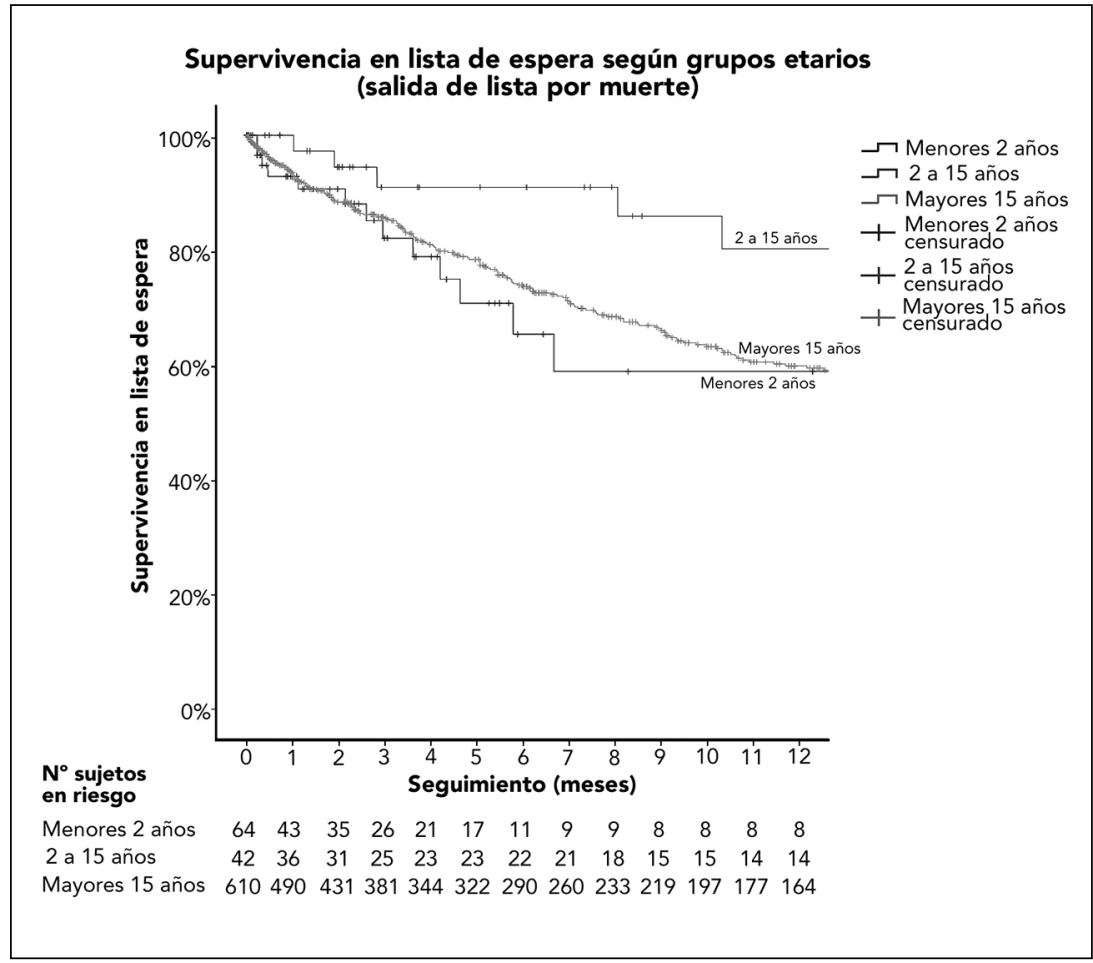

Figura 2. Curvas de supervivencia en LE programa TH por hepatopatías crónicas: Comparación de la supervivencia en LE entre pacientes pediátricos y adultos.

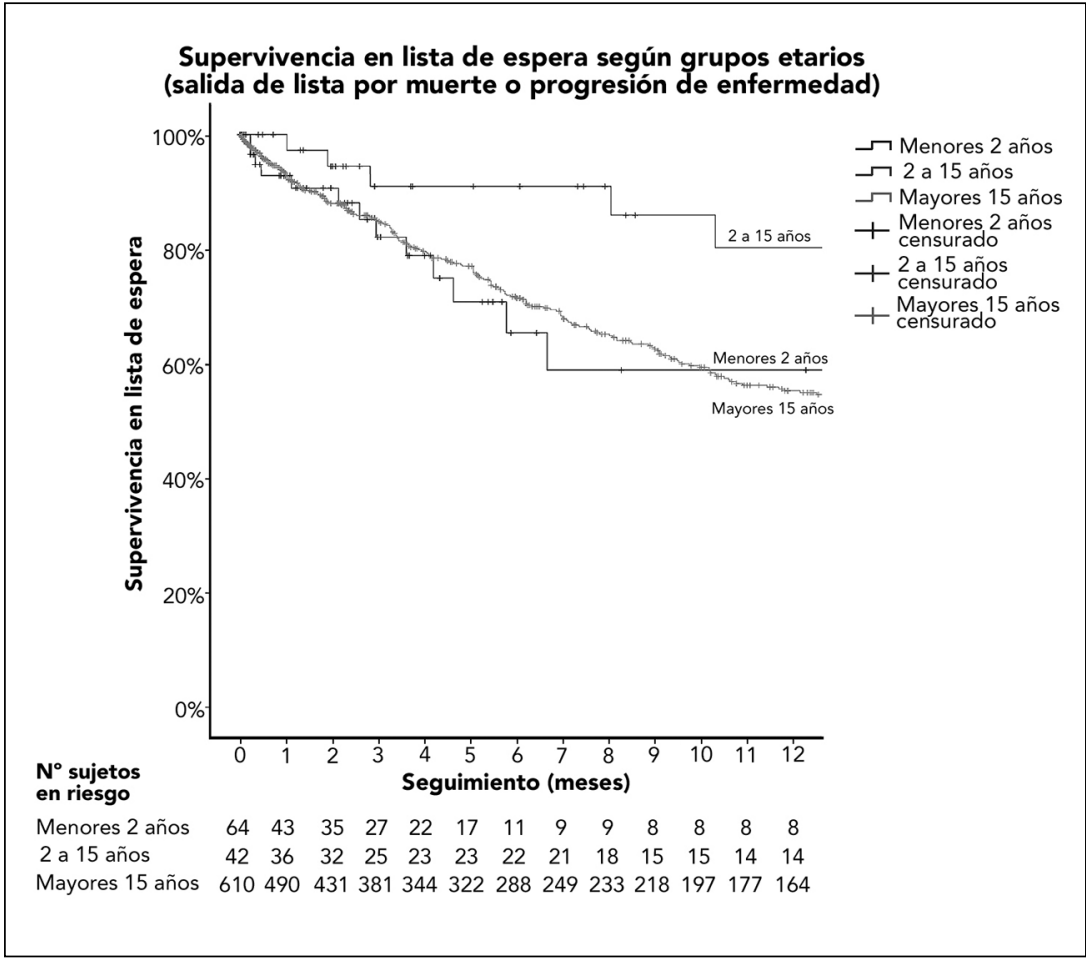

Figura 3. Curvas de supervivencia en LE programa TH por hepatopatías crónicas: Comparación de la tasa de salida de lista entre pacientes pediátricos y adultos. 
Supervivencia post trasplante según grupos etarios

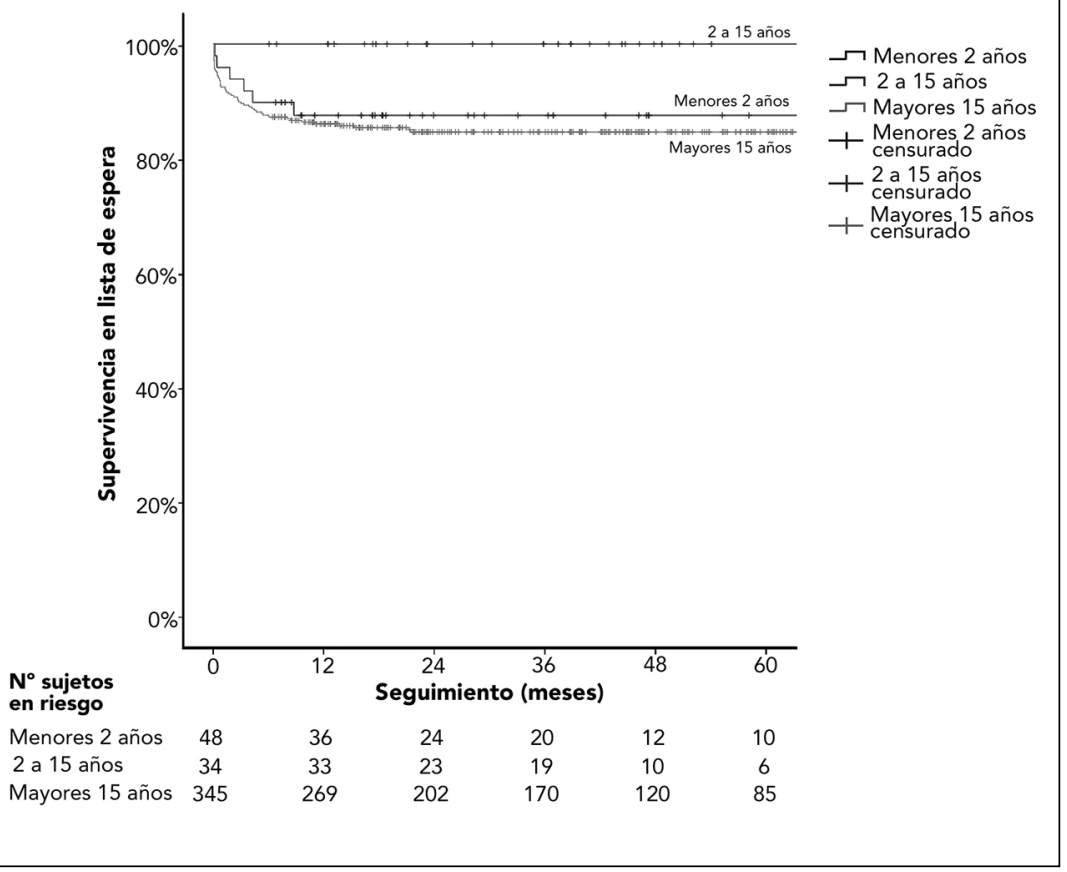

Figura 4. Supervivencia postrasplante en candidatos con hepatopatías crónicas según grupo etario.

Supervivencia post trasplante según tipo de trasplante (menores 15 años)

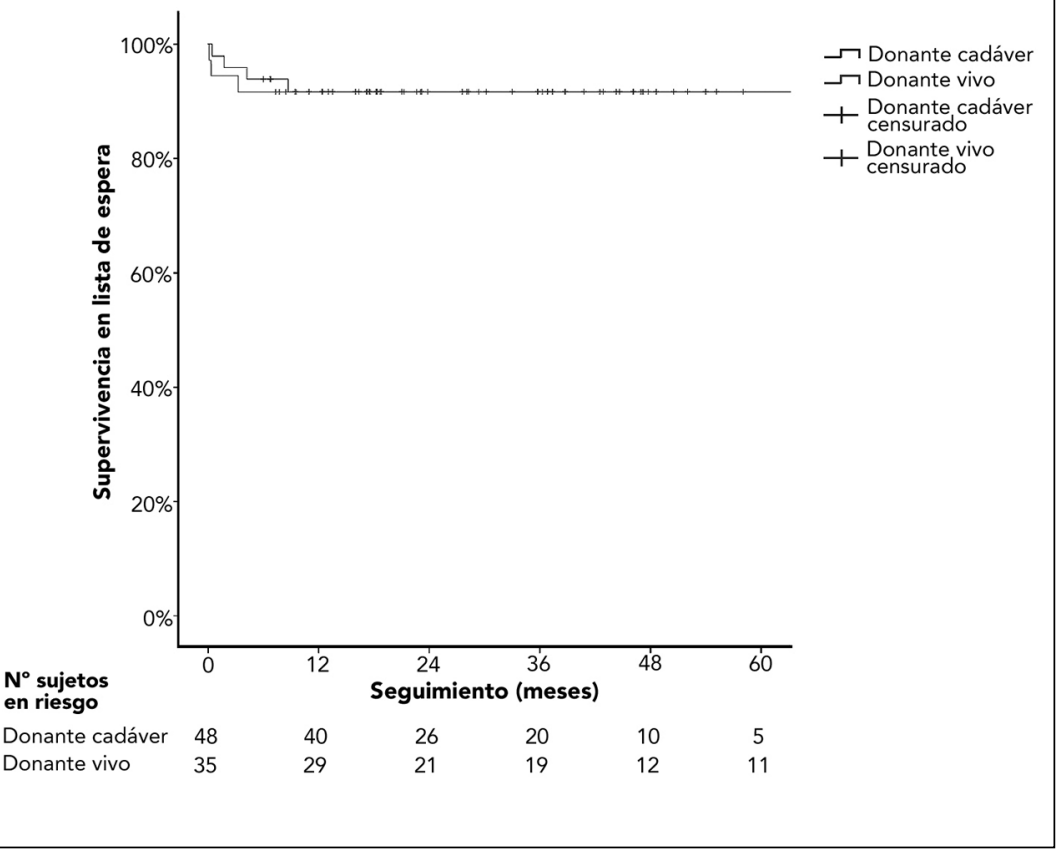

Figura 5. Supervivencia postrasplante en candidatos menores de 15 años con hepatopatías crónicas según tipo de injerto (donante cadáver versus donante vivo). 


\section{Discusión}

Nuestro estudio demuestra que existe inequidad en el acceso a un trasplante cadavérico en pacientes pediátricos menores de 2 años alistados por hepatopatías crónicas, basado en el actual sistema de priorización MELD/PELD, siendo este trabajo el primero en describir esta situación en Chile. Las principales desventajas en este grupo son: 1 . Menor acceso a un injerto de DC en menores de 2 años; 2. Los pacientes pediátricos alistados por patologías colestásicas tienen aún menor acceso a THDC que los alistados por otras patologías; 3 . Aunque los pacientes pediátricos y adultos tengan tiempos de espera similares para el THDC, la mortalidad en LE se produce antes en candidatos pediátricos; 4 . Los menores de 2 años tienen una mortalidad estimada en LE al año mayor que cualquier otro grupo etario $(38,1 \%)$.

Dada la alta mortalidad en lista de espera, especialmente en menores de 2 años, los equipos tratantes se ven obligados a buscar otras opciones como el THDV, especialmente en pacientes pediátricos. Aunque el acceso a THDC es similar al comparar población pediátrica con adultos (41 vs $47,8 \%$ ), este fue considerablemente menor en el grupo de menores de 2 años versus 2-15 años ( $27,9 \%$ vs $57,4 \%$, respectivamente). La principales causas de alistamiento en pacientes pediátricos son colestásicas (50\%), lo que es concordante con lo encontrado en series internacionales ${ }^{9,10}$. Estas causas no ven reflejada su mortalidad de acuerdo al MELD/PELD y actualmente no están consideradas dentro de las excepciones para puntaje extra en nuestro país. De hecho, el puntaje MELD/PELD al alistamiento fue significativamente menor en los pacientes pediátricos comparados con otras etiologías. Esto podría explicar que solo 17,9\% de los menores de 2 años alistados por etiología colestásica alcanzaron un THDC y en el grupo de 2 a 15 años $20,7 \%$.

Los menores de 15 años fallecen antes que los adultos en LE. Aunque el THDV ha tenido un impacto en paliar esta mortalidad, no se considera la primera alternativa de tratamiento en candidatos pediátricos. En efecto, la opción del DV tiene otras implicancias, como el riesgo de morbilidad al que se somete a un donante previamente sano y la dificultad para contar con un donante que cumpla con los requisitos necesarios, como tener un estado nutricional y de salud compatible, espe- cialmente debido a la gran prevalencia de obesidad $(34,4 \%)$ y de hígado graso no alcohólico $(25,24 \%)$ en la población chilena ${ }^{11}$. También existen otras barreras a este tratamiento, como las adicciones, la anatomía desfavorable, el temor a las complicaciones quirúrgicas y costos económicos ${ }^{12}$.

A pesar de contar con THDV, la mortalidad en LE de pacientes pediátricos a un año es $25,1 \%$. De hecho, aquellos pacientes que fallecieron lo hicieron precozmente, ya que la mitad de los pacientes pediátricos fallecen antes de cumplir 3 meses en LE. Por ende, la implementación del sistema MELD/PELD en Chile no ha disminuido desde la era previa a MELD/PELD (25\%-29\%), lo cual aún está por encima de cifras latinoamericanas de mortalidad pediátrica en LE (Argentina $18 \%$ y Brasil $15 \%)$ o norteamericanas $(10 \%)^{7,13,14}$. Sin duda, esto genera una gran cantidad de años de vida potencialmente perdidos en nuestros candidatos.

Creemos prioritario desarrollar estrategias para aminorar esta inequidad en la LE para TH en Chile. La tasa de donación chilena alcanza 7 donantes por millón de habitantes, con una negativa familiar de $51 \%{ }^{15}$. Esta cifra es claramente inferior al comparar contra otros países como España (30 donantes por millón de habitantes) ${ }^{6}$. Por lo tanto, la elevada salida de lista de espera podría ser parcialmente atribuida a nuestra baja tasa de donantes. Una opción para subsanar el escaso acceso al injerto cadavérico en todos los candidatos menores de 15 años es aumentar el puntaje de los candidatos pediátricos, como lo hacen en Brasil, donde multiplican el puntaje PELD por 3, como en la serie de Neto y cols. en Sao Paulo, que demostró un aumento de $62 \%$ en el número de trasplantes con DC realizados en candidatos menores de 12 años, así como también una reducción significativa en el tiempo de acceso al trasplante, ya que con esta medida lograron que los candidatos pediátricos quedaran mejor ubicados en la LE' ${ }^{16}$.

En Chile, se ofrecen los donantes a receptores de acuerdo al tamaño y compatibilidad de grupo. Como la mayoría de los donantes son adultos, estos se ofrecen principalmente a adultos. Una opción para mejorar el acceso a injertos de DC en candidatos pediátricos es aumentar los split hepáticos, en que un hígado de un DC adulto se divide en dos partes, un segmento izquierdo más pequeño, adecuado para un receptor pediátrico 
y un segmento derecho más grande, para un receptor adulto o pediátrico ${ }^{17}$. En este contexto es destacable la experiencia descrita en Birmingham, Reino Unido, por Battula y cols., en que muestran el rendimiento de los trasplantes split en un centro de trasplante pediátrico, antes y después de la implementación de una política llamada "Intención de dividir", posterior a lo cual vieron un aumento significativo en este tipo de procedimientos, de $7 \%$ a $18 \%$, con lo que lograron cubrir con injertos provenientes de un split hasta $65 \%$ de los trasplantes pediátricos realizados durante el periodo estudiado. Además, lograron una mortalidad en lista de espera de $0 \%$ en los últimos 4 años del estudio $^{18}$. No obstante, el split se ha utilizado de forma muy escasa en Chile, especialmente por sus dificultades técnicas en las anastomosis vasculares y biliar ${ }^{19}$. Por lo tanto, es necesario capacitar adecuadamente a los equipos quirúrgicos y contar con un sistema que asegure el traslado de los injertos una vez divididos, a fin de promover esta técnica de forma segura.

Creemos que es necesario reevaluar la puntuación MELD/PELD asignada a los pacientes menores de 15 años alistados por colestasia y, especialmente, a todos los pacientes menores de 2 años, independiente de la causa de alistamiento, para optimizar su acceso al injerto cadavérico y contribuir a evitar las muertes en LE.

En cuanto a las limitaciones del estudio, es importante destacar que la obtención de datos fue retrospectiva y utilizando datos anónimos codificados con la identificación del ISP, lo que limitó el análisis del puntaje al momento del trasplante y las causas de muerte. Asimismo, se identificó que $57,1 \%$ de los pacientes pediátricos se alistó con algún tipo de excepción, lo cual es similar a lo identificado previamente en adultos chilenos (57,9\% de excepciones), sin embargo, no se logró precisar la causa específica de excepción ${ }^{6}$. A futuro, sería interesante evaluar aspectos sociodemográficos, clínicos y de laboratorio que permitan identificar otros factores que aumentan la inequidad en población pediátrica. Esperamos continuar evaluando la importancia del MELD/ PELD operacional en la estadía en LE de nuestros pacientes y como las excepciones modifican la equidad de esta, junto con identificar los motivos que llevan a perder injertos ya procurados, con el fin de poder instaurar medidas que maximicen el uso de los injertos a nivel nacional.

\section{Conclusión}

En Chile existe una elevada mortalidad en lista de espera para trasplante hepático donante cadáver. El acceso a injertos de DC es significativamente menor en los candidatos menores de 2 años alistados por todas las causas crónicas, con una elevada mortalidad en lista de espera. Las etiologías colestásicas son la principal causa de alistamiento en menores de 15 años, y tienen una menor probabilidad de recibir THDC, especialmente en menores de 2 años. Es indispensable establecer nuevas políticas públicas para incrementar la tasa de donantes y mejorar la equidad del sistema actual de priorización.

\section{Referencias}

1. Alonso FT, Garmendia ML, Aguirre M, Searle J. [Mortality trend from liver cirrhosis in Chile from 1990 to 2007]. Rev Med Chile 2010; 138 (10): 1253-8.

2. Benítez C, Wolff R. Current Status and Future Challenges of Liver Transplantation Programs in Chile. Liver Transpl 2018; 24 (12): 1757-61.

3. Hepp J, Zapata R, Buckel E, Martínez J, Uribe M, Díaz JC, et al. [General considerations, indications and contraindications for liver transplantation in Chile: a multicenter consensus development document]. Rev Med Chile 2008; 136 (6): 793-804.

4. Malinchoc M, Kamath PS, Gordon FD, Peine CJ, Rank J, ter Borg PC. A model to predict poor survival in patients undergoing transjugular intrahepatic portosystemic shunts. Hepatology (Baltimore, Md) 2000; 31 (4): 86471.

5. McDiarmid SV, Anand R, Lindblad AS. Development of a pediatric end-stage liver disease score to predict poor outcome in children awaiting liver transplantation. Transplantation 2002; 74 (2): 173-81.

6. Díaz LA, Norero B, Lara B, Robles C, Elgueta S, Humeres $\mathrm{R}$, et al. Prioritization for liver transplantation using the MELD score in Chile: Inequities generated by MELD exceptions: A collaboration between the Chilean Liver Transplant Programs, the Public Health Institute and the National Transplant Coordinator. Ann Hepatol 2019; 18 (2): 325-30.

7. Dip M, Cejas N, Cervio G, Villamil F, Tagliafichi V, Hansen Krogh D, et al. Results after the adoption of a MELD/PELD-based liver allocation policy in Argentina. Pediatr Transplant 2015; 19 (1): 56-61.

8. Hsu EK, Shaffer ML, Gao L, Sonnenday C, Volk ML, 
Bucuvalas J, et al. Analysis of Liver Offers to Pediatric Candidates on the Transplant Wait List. Gastroenterology. 2017; 153 (4): 988-95.

9. ELTR. European Liver Transplant Registry: Results. Acceso el 16 de agosto de 2019 [Available from: http:// www.eltr.org/-Results-.html.

10. Kasahara M, Sakamoto S, Fukuda A. Pediatric living-donor liver transplantation. Semin Pediatr Surg 2017; 26 (4): 224-32.

11. MINSAL. Primeros y segundos resultados de la Encuesta Nacional de Salud 2016-2017-2018 [Available from: http://epi.minsal.cl/resultados-encuestas/.

12. Uribe MM, Hunter MB, Alba GA. Trasplante hepático pediátrico estudio descriptivo de la experiencia recogida por el grupo de trasplante pediátrico de Clínica Las Condes y hospital Luis Calvo Mackenna. Rev Med Clin Las Condes 2010; 21 (2): 254-65.

13. Feier F, Antunes E, D'Agostino D, Varela-Fascinetto G, Jarufe N, Patillo JC, et al. Pediatric liver transplantation in Latin America: Where do we stand? Pediatr Transplant 2016; 20 (3): 408-16.

14. Perito ER, Mogul DB, VanDerwerken D, Mazariegos G,
Bucuvalas J, Book L, et al. The Impact of Increased Allocation Priority for Children Awaiting Liver Transplant: A Liver Simulated Allocation Model (LSAM) Analysis. J Pediatr Gastroenterol Nutr 2019; 68 (4): 472-9.

15. Arriagada AM, Maquilón S, Benítez C. [Organ Transplantation in Chile]. Rev Med Chile 2018; 146 (5): 6756.

16. Neto JS, Carone E, Pugliese RP, Fonseca EA, Porta G, Miura I, et al. Modified pediatric end-stage liver disease scoring system and pediatric liver transplantation in Brazil. Liver Transpl 2010; 16 (4): 426-30.

17. Toso C, Ris F, Mentha G, Oberholzer J, Morel P, Majno P. Potential impact of in situ liver splitting on the number of available grafts. Transplantation 2002; 74 (2): 222-6.

18. Battula NR, Platto M, Anbarasan R, Perera MT, Ong E, Roll GR, et al. Intention to Split Policy: A Successful Strategy in a Combined Pediatric and Adult Liver Transplant Center. Ann Surg 2017; 265 (5): 1009-15.

19. Hackl C, Schmidt KM, Süsal C, Döhler B, Zidek M, Schlitt HJ. Split liver transplantation: Current developments. World J Gastroenterol 2018; 24 (47): 5312-21. 